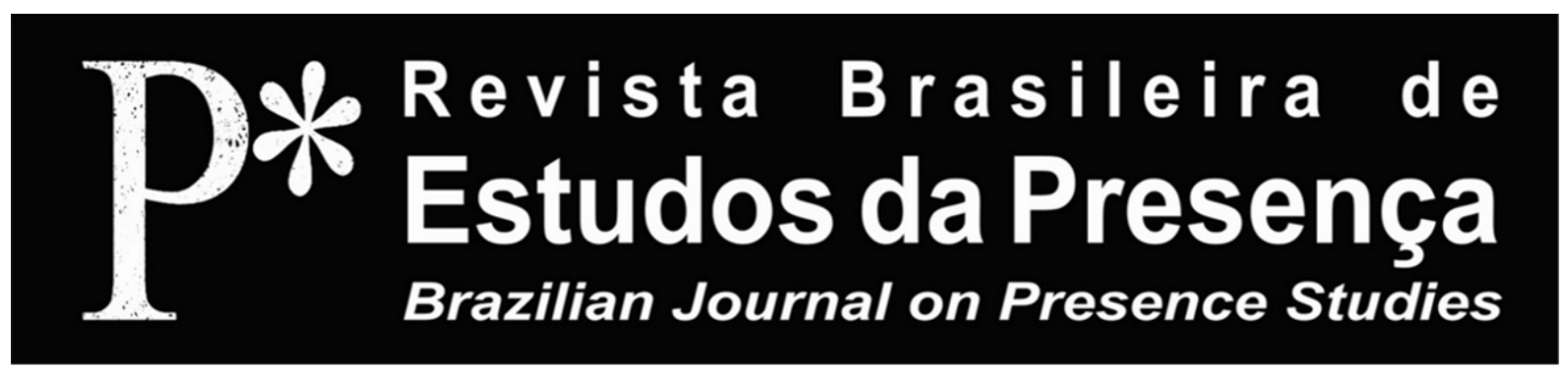

ISSN 2237-2660

\title{
Pour une Écologie des Somatiques?
}

\author{
Joanne Clavel \\ Museum National d'Histoire Naturelle - Paris, France \\ Isabelle Ginot \\ Université Paris 8 Vincennes Saint-Denis - Paris, France
}

RÉSUMÉ - Pour une Écologie des Somatiques? - Ce texte traite de la parenté conceptuelle et théorique entre Somatiques et Écologie scientifique. Après une définition de la notion scientifique d'écologie, il s'attache à définir trois notions clés de l'écologie: celle de potentiel, qui permet de décrire de façon innovante le modèle de relation entre sujet et environnement au sein des Somatiques, et celles de diversité et de réciprocité, à partir desquelles l'article se veut une invitation et un programme à penser l'intégration des Somatiques au paradigme écologique scientifique, et en appelle aux acteurs somatiques pour transformer leurs pratiques selon une éthique environnementale. Mots-clés: Somatiques. Écologie. Potentiel. Diversité. Réciprocité.

ABSTRACT - For an Ecology of Somatics? - This text studies notions that are common to Somatics and Scientific Ecology. After defining the scientific notion of Ecology, this essay studies three key-notions in Ecology: the concept of potential, which allows an innovative description of the relationship subject/environment in Somatics, and the notions of diversity and reciprocity, with which this essays suggests that Somatics could be integrated into the Ecological paradigm, and calls the Somatics actors to transform their practices according to Environmental ethics.

Keywords: Somatics. Ecology. Potential. Diversity. Reciprocity.

RESUMO - Por uma Ecologia da Somática? - Este texto trata do parentesco conceitual e teórico entre Somática e Ecologia Científica. Após definir a noção científica de Ecologia, procuram-se definir três conceitos-chave da Ecologia: o de potencial, que permite descrever, de forma inovadora, o modelo de relaçáo entre sujeito e meio ambiente no âmbito da Somática; e os de diversidade e reciprocidade, a partir dos quais o artigo se revela como um convite e um programa para se pensar a respeito da integração da Somática ao paradigma ecológico científico. Para tal, recorre-se aos atores somáticos para transformar suas práticas de acordo com uma ética ambiental.

Palavras-chave: Somática. Ecologia. Potencial. Diversidade. Reciprocidade. 
Nous voudrions, dans cet essai, esquisser quelques balises pour inviter chercheurs et praticiens à investiguer un champ encore peu travaillé, celui des Somatiques en relation avec l'écologie, et plus précisément, avec le paradigme scientifique de l'écologie. Le champ est vaste, mais il nous importe, dans la mesure où il pourrait permettre de développer de nouvelles approches de la pensée écologique, mais aussi (surtout?) de nouvelles conceptualisations et de nouveaux usages des Somatiques. Il nous semble tout d'abord qu'il existe une sorte de parenté informelle dans la pensée des Somatiques et de l'écologie, sans que cette parenté ait jamais véritablement été interrogée. Mais il nous semble aussi que le paradigme scientifique de l'écologie constitue aujourd'hui une ressource politique qui devrait stimuler et inquiéter les pratiques somatiques pour un engagement politique autre, et telle est l'invitation, ou le programme, que nous souhaitons lancer aux chercheurs et praticiens des deux domaines.

Dans un premier temps, nous reviendrons sur les différents usages du terme écologique qui se diffuse rapidement dans différentes disciplines et plus particulièrement dans les sciences humaines qui travaillent sur la question de la perception. Comme tout terme à la mode, son apparition exige de s'interroger sur la teneur et l'implication de son utilisation et, éventuellement, de ses usages impropres. Dans un second temps, nous présenterons une première notion, celle de potentiel, qui fonde notre hypothèse initiale d'une communauté de pensée entre Somatiques et écologie. Enfin, nous appellerons les acteurs des Somatiques à réfléchir à ce que pourrait être une utopie écosomatique, ou un cadre théorique écologique inspiré directement des sciences de l'écologie et de ses implications philosophiques suivant les éthiques environnementales contemporaines, à partir de deux autres notions écologiques cruciales: celles de diversité et de réciprocité.

\section{L'Hypothèse Initiale}

Les Somatiques définissent l'individu, d'une part comme global - un peu plus que la somme des substances anatomique, consciente, inconsciente et énergétique - et d'autre part comme indissociable de son environnement. Cette approche holistique est un pilier fondamental dans la diversité des techniques somatiques qui existent. Par ailleurs, les termes d'environnement, de milieu, et de nature - entre autres - sont prégnants dans les discours des pratiques somatiques, 
ainsi que dans nombre de leurs références théoriques (notamment celles de la phénoménologie et de la psychologie). Plus récemment, le terme d'écologie a été rapproché des Somatiques, et le terme de somatiques éco-centrées a même pu être proposé par Fortin. Enfin, les Somatiques émergeant en réponse aux dégradations environnementales se rapprochent par là du souci environnemental. Cette constellation d'observations semble permettre d'intégrer d'emblée les Somatiques dans un cadre de pensée écologique, et impose d'activer un point de vue critique sur l'usage des concepts proprement écologiques. De quelle écologie parlent les Somatiques? Nous proposons donc de revenir sur quelques fondamentaux de ce paradigme en présentant une lecture à partir d'une pratique somatique: la méthode Feldenkrais. Cette méthode, du nom de son fondateur Moshe Feldenkrais, est parmi les plus répandues des pratiques somatiques. Elle a pour objet l'amélioration des coordinations (ou automatismes acquis) et se pratique en séances collectives ou individuelles. Chaque séance est dédiée à un mouvement précis, souvent simple et quotidien (s'asseoir, rouler, se lever, attraper etc.), décliné, déconstruit dans d'innombrables variations qui conduisent progressivement à intégrer un répertoire gestuel profondément affiné, et surtout, selon les termes du fondateur, un répertoire plus large et ouvert d'adaptations au milieu. Comme toutes les pratiques somatiques, la méthode Feldenkrais s'appuie sur un travail très raffiné de prise de conscience, autrement dit, sur la multiplication d'activités perceptives très élaborées, précises et diversifiées, qui nous ont fait ailleurs parler "[...] d'érudition perceptive» (Ginot, 2013, p. 22), et qui situent d'emblée le cœur de la pratique dans les échanges entre le sujet et son milieu. Dans ses ouvrages, Feldenkrais ne cesse de revenir à sa définition du mouvement non pas comme action corporelle, mais comme interaction avec le monde. Ses écrits - qui visent plus à tenter de construire une théorie générale qu'à véritablement décrire et définir sa pratique - insistent ainsi sur les diverses natures de milieux qui forment l'environnement humain. Carla Bottiglieri montre comment une dimension importante de la pratique Feldenkrais consiste à intervenir sur le milieu, et non sur le corps, pour permettre l'émergence de nouvelles coordinations, en intervenant sur les appuis et en modifiant les supports notamment (Bottiglieri, 2015). C'est donc tant sur la théorie de Feldenkrais, que sur sa méthode, que nous fondons notre première hypothèse de la pertinence du paradigme écologique. 


\section{L'Écologie du Geste est-elle Écologique?}

C'est principalement par le biais de la perception, de ses définitions théoriques comme de ses usages pratiques, que les Somatiques rencontrent le paradigme écologique. La perception, comprise comme la variété des échanges entre le sujet et son environnement, est en effet au cœur de toutes les Somatiques et de leurs diverses techniques de développement du geste et de la posture. Elle est aussi un objet central de la phénoménologie. Peu présente de façon explicite dans les discours des fondateurs, celle-ci constitue cependant un substrat théorique important dans la mesure où les Somatiques accordent une place centrale au sentir et à l'expérience à la première personne, selon l'expression de Thomas Hanna (1995). Merleau-Ponty ${ }^{1}$ a notamment insisté sur la continuité entre moi et le monde, posant ce qui pourrait se présenter de prime abord comme une pensée écologique. Pourtant, si la phénoménologie pense l'interaction, celle-ci est centrée sur l'expérience personnelle mais ne fait pas de place à la réciprocité. Surtout, la phénoménologie n'envisage que le point de vue des humains. Il n'est donc pas possible dans le cadre phénoménologique de décrire et théoriser les conditions de l'interaction entre entités ou êtres vivants et leurs environnements abiotiques.

L'écologie perceptive, ouverte par les travaux de James J. Gibson et sa notion d'affordance, est un autre des cadres de référence des Somatiques. L'affordance, ce serait l'ensemble de toutes les possibilités d'actions offertes par un environnement qui, bien que considérées comme objectives, doivent toujours être mises en relation avec l'acteur qui va les percevoir en fonction des usages gestuels que lui ouvre cet environnement. Ainsi, la feuille de nénuphar peut être perçue comme support potentiel par une grenouille, tandis qu'un poisson la perçoit plutôt comme pourvoyeuse d'ombre. Cependant, dès l'introduction de The Ecological Approach to Visual Perception (1979), Gibson écarte de l'expérience perceptive les êtres vivants appartenant à un autre règne que celui de l'animal, proposant au passage de considérer les plantes comme inertes: «De façon générale, les plantes ne sont pas animées; elles ne bougent pas, ne connaissent pas de comportements, n'ont pas de système nerveux, ni de sensations. De ce point de vue elles sont donc comme les objets de la physique, de la chimie et de la géologie ${ }^{2} »$ (Gibson, 1986, p. 7).

De plus, Gibson ne propose pas de penser l'interaction de façon réciproque: dans son modèle, apparaît systématiquement une 
prépondérance d'un des acteurs, celui du je, celui du point de vue. Si expérimenter le monde et interagir avec lui sont intimement liés, cette complète asymétrie de l'interaction fait apparaître un rapport utilitariste pour l'un des deux acteurs: jamais la relation n'est pensée comme autre chose que la somme des deux parties. La seule valeur qui lui soit attribuée est une valeur d'usage, une valeur instrumentale: celle du service qui est rendu à l'acteur de l'interaction, celui qui s'énonce par le je.

Toute une tradition moderne a cherché à introduire une philosophie du nous:

Au fond de l'image il y a l'imagination et au fond de l'imagination il y l'autre, la vue de l'autre, c'est à dire la vue sur l'autre et l'autre en tant que vue - qui souvre aussi, par conséquent, en tant quautre de la vue, non-vue prévoyante. L'autre me fait vis-à-vis et ainsi se montre en tant quautre. L'image est tout d'abord autre et de l'autre, altérée et altérante. Elle donne l'autre selon lequel le même peut être montré (Nancy, 2003, p. 176).

Cependant, cette ouverture à l'altérité se fonde sur une communauté excluant les non-humains. Le point de vue de l'écologie impose de poser cette question: au-delà des intérêts directs personnels ou sociaux que l'on peut tirer des interactions sociales, dans quelle mesure pouvons-nous ouvrir le nous de l'interaction aux non-humains? D'une part, la biologie du $21^{\text {ème }}$ siècle nous enseigne que la subjectivité et l'intersubjectivité sont partagées par de nombreux non-humains (céphalopodes, oiseaux, mammifères etc.). D'autre part, au-delà des avancées récentes de la biologie, il s'agit de penser les enjeux éthiques et moraux de l'interrelation: l'homme continuera-t-il à considérer toutes les autres formes de vie qui peuplent le monde comme des moyens à sa disposition, ou au contraire, toutes ces formes de vie peuventelles être considérées comme une valeur de non usage, disposent-t-elles d'une valeur intrinsèque? Un chien Bas-rouge a-t-il une valeur intrinsèque en dehors de la protection de la maison qu'il assure? Le gypaète barbu a-t-il une valeur intrinsèque en dehors de l'observation ornithologique? L'écosystème de la montagne Sainte-Victoire, objet de célèbres peintures de Cézanne, at-elle une valeur intrinsèque en dehors de son importance dans le monde de l'art? Ce double ancrage - dans le point de vue scientifique et dans le débat philosophique - est partagé par l'écologie et les Somatiques, celles-ci mêlant, dans leurs textes comme dans leurs pratiques, les références aux savoirs scientifiques de leur temps et une approche éthique de leur pratique.

Les recherches en phénoménologie, comme en écologie perceptive, tout en se focalisant sur l'interaction, laissent donc échapper le 
point central du paradigme écologique, à savoir la réciprocité de la relation, que l'on pourrait nommer relation éco-centrique ou biocentrique $^{3}$. Pourtant, ces deux cadres de l'affordance et de la phénoménologie peuvent nourrir la conceptualisation de l'expérience, de la rencontre et de l'ouverture à l'autre. Plutôt que de les exclure de notre corpus de réflexion, nous voudrions essayer d'élargir leurs perspectives vers un éco-centrisme en y incluant la communauté plus large des non-humains. L'abondance des références aux connaissances scientifiques du vivant présent dans les écrits des Somatiques ${ }^{4}$ nous y invite et cette décentralisation est fondamentale pour penser notre environnement et nos interactions avec lui. Pour cela notre proposition consiste à considérer les pratiques somatiques comme des méthodes travaillant un ensemble de potentiels que l'histoire évolutive des espèces donne à partager et qui façonne en retour l'environnement.

\section{Une Approche Scientifique de l'Écologie}

Les textes somatiques font massivement référence aux sciences et revendiquent tout particulièrement un ancrage au sein de l'évolution du vivant. Par exemple, tous les écrits de Moshe Feldenkrais ont un passage consacré à la théorie de l'évolution. Il a lu L'Origine des Espèces (Darwin, 1985) et cultive une profonde admiration pour Charles Darwin. De même, cet héritage du passé de l'évolution du vivant qui réside en chacun de nous est au cœur de la pratique du Body-Mind Centering. La biologie nous apprend que ce bagage partagé entre toutes les espèces, ce commun du vivant, est plus ou moins grand selon le degré de parenté. Ainsi je partage au moins l'alphabet du vivant avec n'importe quelle bactérie, une respiration cellulaire presque identique avec les amibes, et quasiment tout mon héritage avec mon proche cousin le chimpanzé (même si cet écart minimal donne un résultat étonnamment différent!) Retracer ces liens de parenté entre les différentes entités vivantes est l'objet de la phylogenèse. On l'étudie également à une plus petite échelle, généralement fonctionnelle, par l'étude du développement embryonnaire: l'ontogenèse. On observe en effet une très grande similitude développementale dans les premiers stades embryonnaires chez la grenouille, le poulet ou l'homme, trace de cet héritage commun mobilisé lors des premières étapes de la vie. Beaucoup de textes théoriques des Somatiques font référence à ces processus onto- et phylogénétiques, et c'est particulièrement le cas des écrits de Feldenkrais et de Bonnie B. Cohen 5 . 


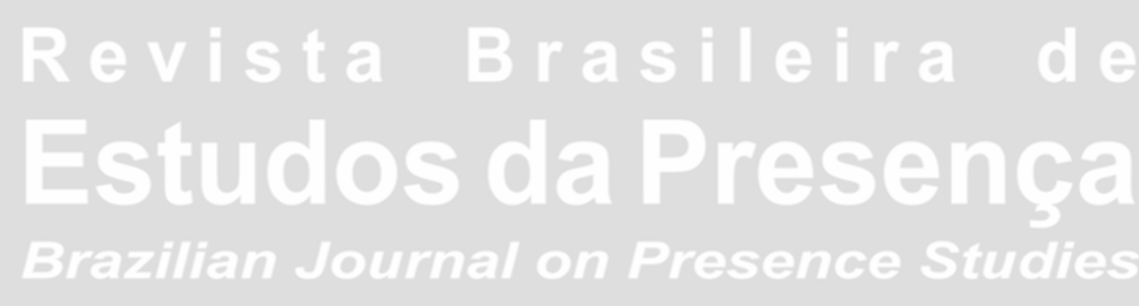

En quoi aujourd'hui la phylogenèse et l'ontogenèse nous permettent-elles de mieux comprendre ce tout? Si le commun perceptif entre l'homme et le mouton est bien plus grand que celui que partagent le mouton et la mouche, l'étendue perceptive n'en est pas moins contrainte par des processus biologiques à différentes échelles intégratives. En effet, le domaine des possibles est contraint par ce commun hérité - le bagage évolutif - mais également par les particularités du développement individuel. Les variations apparues au sein de la phylogenèse ont deux propriétés: leur création est aléatoire (les mutations) et leur expression est dépendante de l'environnement. De plus, le maintien de ces variations dépend soit d'une expression sélectionnée par le processus d'adaptation, soit d'une expression neutre qui se maintient par dérive, par tirage aléatoire ${ }^{6}$. Mais, lorsqu'elles relèvent de la phylogenèse, les contraintes de l'expérience sont invariables pour la temporalité de l'expérience ou presque. En effet, ce sont ces mêmes contraintes que l'on retrouve lors de l'ontogenèse d'un individu. Or, leur expression est la conséquence d'une interaction avec l'environnement. Plus précisément cette interaction se joue entre d'une part, l'héritage génétique et d'autre part, l'environnement dans lequel se trouve l'individu, et les interactions moléculaires fortuites au sein de chacune de ses cellules. À cette échelle d'étude, les contraintes phylogénétiques délimitent un domaine du possible pour la performance des cellules:

La cause de comportement asynchrone des cultures bactériennes tient à la distribution aléatoire des différents types de molécules dans les cellules filles au moment de la division cellulaire. Chaque cellule fille, pour produire les nouvelles populations de molécules nécessaires à une nouvelle division, mettra ainsi un temps différent; [...] de tels processus aléatoires doivent expliquer un grand nombre de variations entre organismes, y compris des variations au niveau du système nerveux central (Lewontin, 2003, p. 48).

Transposé à l'individu humain, cet exemple permet de comprendre que les réalisations du sujet ne doivent pas être pensées en termes de capacités inhérentes au sujet, mais en termes de potentiel, qui s'actualise pour chacun dans un moment présent. Les interactions, ou la co-construction entre le gène, l'environnement et la performance moléculaire sont singulières: elles ne peuvent être prévues ni à partir du seul environnement, ni à partir du seul génotype, ni à partir de la seule performance. De plus, la notion de potentiel permet de considérer l'échelle évolutive et le dépassement du domaine des 
possibles sur du long terme - sur des centaines de générations. En 1973, Leigh Van Valen ${ }^{7}$ soulève un paradoxe de l'évolution et propose pour y remédier ce que l'on nomme aujourd'hui l'hypothèse de la Reine Rouge, en référence à l'ouvrage de Lewis Carroll De l'autre Côté du Miroir. L'environnement ne cesse de changer, de sorte que l'adaptation à l'environnement d'hier ne donne aucun avantage pour survivre à celui de demain. Ainsi, telle la Reine des Échecs qui comprit qu'elle devrait continuer à courir afin de rester sur place puisque le sol bougeait sous ses pieds, les organismes doivent être dans une course sans fin pour s'épanouir dans leur environnement. Le tout est redonné à travers l'environnement et l'individu dans un balancement entre le passé (son histoire évolutive) et la projection (son devenir). La compréhension du développement de la vie nous permet de baliser et de définir les contours de l'expérience du temps présent, que nous nommerons présence. Ce processus de développement, cette coévolution à différentes échelles de temps (temps évolutif, temps ontogénique, temps socioculturel, temps personnel) conditionne notre expérience vécue, ainsi contrainte par les attributs de l'histoire évolutive qui serpente dans la contingence de l'espace des potentiels.

\section{La Notion de Potentiel dans la Méthode Feldenkrais}

Absente des discours théoriques de Moshe Feldenkrais, la notion de potentiel semble pourtant précisément décrire la construction du changement et ses aléas au sein d'une séance. La structure de la séance Feldenkrais individuelle (où le praticien intervient surtout par le toucher auprès de son élève) comme de la séance collective (où il propose au groupe un ensemble de variations guidées verbalement) a pu être analysée comme une intervention par l'environnement. Le praticien de la méthode Feldenkrais n'intervient pas sur le corps de son élève, mais fait plutôt varier des éléments de son environnement: par l'organisation de contraintes spatiales et gravitaires très spécifiques, en proposant par le toucher ou l'indication verbale l'exploration de variations des appuis au sol, des directions dans l'espace, la séance Feldenkrais se présenterait comme une modification temporaire de l'environnement visant à susciter un changement durable dans les interactions du sujet (de l'élève) avec cet environnement, et tout particulièrement d'un élément invariant fondamental: la force gravitaire.

La notion de potentiel permet de comprendre une dimension importante de la méthode Feldenkrais et de l'ensemble des pratiques 
somatiques, qui les distingue radicalement de formes médicales occidentales classiques et les rapproche - en partie - du paradigme écologique. Tout d'abord, comme Feldenkrais y insiste dans ses écrits, la séance repose sur la non-séparation du sujet et de son environnement: pour Feldenkrais, l'environnement fait partie intégrante du sujet, ce qui explique cette construction de la pratique par constitution d'un environnement provisoire, favorable à la réorganisation des habitudes d'interaction. Ensuite, la pratique se construit sur la prise en compte de la contingence de cette interaction. Dans une séance, les indications (verbales pour une séance collective, principalement tactiles et directionnelles dans la séance individuelle) construisent comme une architecture de relations mouvantes avec le sol, avec l'espace, entre différentes parties du corps. Cette architecture compose le schéma de la coordination qui fait l'objet de la séance (par exemple, une rotation de la colonne pour aller regarder derrière soi, ou encore un mouvement du bras et de la colonne pour atteindre un objet imaginaire). Elle vise ainsi, selon les propres termes de Feldenkrais, à restaurer quelque chose de l'idéal optimal du geste, compte tenu des contraintes absolues de l'espèce (éventuellement augmentées des contraintes absolues de l'individu, telles que les malformations structurelles squelettiques ou neuro-motrices). Cependant, le schéma proposé par les indications verbales de la séance collective ne consiste pas à faire quelque chose sur le corps de l'élève, ou encore à rapprocher son corps de l'idéal coordinatif. Le praticien Feldenkrais qui intervient par le toucher auprès de son élève en séance individuelle ne travaille pas sur son corps, et ne prétend pas changer quelque chose à ce corps. Il construit, via ses interventions tactiles ou verbales, une série de changements provisoires du milieu qui constituent autant de variations possibles des relations entre ce milieu et le sujet, et qui dépendent strictement des réponses et interprétations de celui-ci. Ainsi, les effets prévisibles de la séance sont circonscrits dans un spectre de probabilités (l'ensemble de tout ce que l'élève pourrait sentir à l'occasion de cette séance, et dont une partie pourrait constituer un changement plus ou moins durable de son usage du mouvement), mais ne s'actualisent que de façon singulière, laissant intégralement place à l'aléatoire de la rencontre entre le milieu provisoire de la séance, les contraintes du sujet et sa temporalité propre. «C’est la puissance de cette incertitude qu'il faut tout le temps reconquérir, en ce qu'elle arpente le territoire tout en le produisant, elle y inscrit des marges 
de manœuvre, elle suspend l'objectif à un non-savoir de ce devenir [...]» (Bottiglieri, 2013, p. 128).

Car si n'importe quoi ne peut pas advenir (une séance Feldenkrais reste une séance Feldenkrais), le devenir du sujet dans sa rencontre avec cette séance ne peut être totalement prédit ni à partir de la structure du sujet, ni à partir de l'environnement de la séance. En cela, la pratique somatique rejoint le modèle de la Reine Rouge à l'instant présent ${ }^{8}$, et sans doute est-elle un espace d'incarnation ou d'expérimentation in situ de cette hypothèse.

Cette pensée du potentiel rapproche ainsi les Somatiques du paradigme écologique dans la mesure où elle implique une autre pensée temporelle et une autre logique des causes et des effets. Cependant, ce premier niveau d'ancrage écologique ne saurait suffire à inclure les Somatiques dans ce paradigme. Au contraire, il nous impose de poursuivre l'enquête: deux autres notions de l'écologie scientifique nous semblent devoir être pensées par les acteurs somatiques, afin de rêver les transformations somatiques nécessaires à l'intégration au paradigme écologique: celle de diversité et celle de réciprocité.

\section{L'Utopie de la Diversité: pour une Transformation Socio-économique}

Le modèle anthropocentré qui fait de l'homme un être supérieur, et le seul à bénéficier de considérations morales, entre en effet en opposition avec les résultats scientifiques actuels qui considèrent l'environnement non pas comme une toile de fond, mais comme un acteur majeur de l'évolution, tout comme du développement individuel (Malabou, 2007) et du bien-être (Maller et al., 2005). On l'a $\mathrm{vu}$, ce postulat de la suprématie humaine au sein du règne animal est contradictoire avec la reconnaissance d'un héritage partagé avec nos cousins non-humains. Cette filiation commune de tous les êtres vivants, leurs échanges et leurs relations, nous invite expressément à reconsidérer nos devoirs moraux envers les non-humains et à les inviter dans notre vie collective. La remise en cause de la place hégémonique de l'homme impose non seulement d'accueillir les nonhumains dans notre environnement, mais aussi de considérer à son tour l'homme comme un environnement, comme un écosystème accueillant en lui une diversité de non-humains:

Monsieur Lymphocyte parle: Tu n'es qu'un écosystème stupide et égoïste. Tu es fier d'être un animal conscient. Mais 
ce que tu appelles «conscience de toi-même correspond exactement à ma définition de l'égocentrisme... Imagine comment tu concevrais le monde si tu étais à ma place. $\mathrm{Ta}$ sensation d'être un tout, que tu appelles «consciences et qui domine ta perception du monde, $t^{\prime}$ ôte toute lucidité par rapport à la société d'animaux microscopiques qui vivent en toi. Tu nous nommes «cellules` et tu nous considères comme tes prisonniers. Réfléchis un peu... Est-ce que la forêt demande aux animaux et aux plantes qui l'habitent comment ils font pour reconnaître la forêt de la non-forêt afin de défendre son intégrité? (Sonigo; Stengers, 2003, p. 82-83).

Il nous semble que l'abandon du modèle anthropocentré a un double effet sur les usages des Somatiques. D'une part, il faut dès lors s'interroger sur la prise en compte de la diversité humaine elle-même au sein des Somatiques. D'autre part, repenser l'interaction individu/ environnement implique une nouvelle considération de l'individu comme environnement. Il faut alors que la conscience de soi comme tout unique, fondement du champ des Somatiques, s'ouvre également comme une conscience du non-soi, un soma accueillant la vie en son sein, un soma que nous proposerons de nommer soma-écosystème.

Dans les discours et les pratiques somatiques, la représentation de l'humain comme un universel permet aux Somatiques de se dispenser de considérer la diversité au sein de l'humain lui-même. Ainsi, chez Moshe Feldenkrais, malgré l'insistance qu'il met à souligner l'impact de l'environnement culturel (et particulièrement celui de l'environnement linguistique) sur le développement du sujet, il apparaît clairement que l'universel humain qu'il décrit se construit comme occidental, mâle et, admettons-le, issu des classes sociales les plus aisées. Cette universalisation d'une classe sociale et culturelle dominante est un phénomène général des pratiques corporelles qui se développent dans les années 1950 à 1980 . Paradoxalement, alors que la plupart d'entre elles empruntent à des techniques extra-occidentales (yoga, méditation, arts martiaux etc.), elles continuent à regarder ces autres pratiques comme traditionnelles (donc prétendument fixes et primitives). Ainsi, comme cela a pu être décrit à propos de nombre de pratiques et usages corporels de la même époque (Foster, 2011), le paradigme biologique de Feldenkrais produit des effets directement sociaux et idéologiques, en naturalisant et universalisant l'expérience d'un groupe dominant. De plus, ce paradigme théorique est en conflit évident avec les usages de Moshe Feldenkrais lui-même, qui a développé une grande partie de sa pratique à partir de son travail auprès de personnes vivant avec des handicaps physiques, sensoriels, 
moteurs, psychomoteurs, travaillant ainsi à même la diversité que son cadre théorique semblait évincer.

Un autre pendant de l'ouverture à la diversité est à analyser. Les Somatiques participent activement, depuis une vingtaine d'années, à l'essor du marché de la douleur et du bien-être. Cet engagement dans le marché du bien-être est, pour une part, le fait de la marginalisation et de l'exclusion de ces techniques par l'institution des savoirs éducatifs et médicaux (Bottiglieri; Ginot; Salvatierra, 2013). Mais il nous semble aussi être le résultat direct de l'absence de représentation d'une diversité humaine et sociale au sein de l'ensemble humain décrit par les Somatiques. La prise en compte de cette diversité sociale implique une position critique vis-à-vis de l'économie de marché et, également, pour décentrer les pratiques somatiques vers des espaces sociaux autres que ceux du marché du bien-être, la capacité de repenser radicalement certains présupposés pédagogiques, tant ceux-ci sont construits à partir d'une hypothèse d'homogénéité et d'universalité des destinataires des pratiques. Que seraient des Somatiques ouvertes à la diversité humaine, mais aussi au non-humain? Qu'est-ce que cette ouverture ferait aux Somatiques? Que seraient des Somatiques adressées à l'ensemble des soma-écosystèmes du monde du vivant?

\section{L'Utopie de l'Interaction: pour une transformation politique}

Notre deuxième utopie écosomatique emprunte sa question aux implications morales de l'écologie scientifique développées par les éthiques environnementales. C'est parce que le potentiel de l'espèce humaine lui permet tout particulièrement de conscientiser son rapport au monde, et donc d'élaborer des droits et des devoirs envers autrui, qu'il est important de penser la question des réciprocités interactives posée par l'écologie scientifique aujourd'hui et demain, la réciprocité n'impliquant pas une symétrie de la relation. Que font les pratiques somatiques aux individus et à leur environnement, quels sont leurs responsabilités et leurs enjeux?

Dans une version contractualiste des éthiques environnementales, différents modèles cosmopolitiques ont été proposés afin d'élargir l'ensemble des individus envers lesquels nous aurions des obligations morales (par exemple le cosmopolitisme moral et le cosmopolitisme politique). On peut en effet penser une autre forme de lien et de responsabilité entre individus, qui ne se base ni sur une forme de spécisme, ni sur les liens institutionnels que les citoyens ont entre eux mais, comme 
l'a suggéré Aldo Leopold ${ }^{10}$ (1949), sur leur appartenance à des communautés biotiques. La prise de conscience qui fait l'objet des Somatiques peut-elle s'envisager non seulement comme conscience de soi, mais aussi comme conscience du non soi? Et quelle serait la conséquence d'un tel décentrement somatique? Une écosomatique qui s'ouvrirait aux nonhumains pour penser autrement son environnement est-elle possible et quelles en seraient les modalités? Quel serait l'environnement sur lequel interviendrait la séance Feldenkrais, si celle-ci abandonnait le paradigme initial de l'exception humaine? Ce besoin d'une nouvelle éco-citoyenneté engage à repenser les Somatiques dans ce cadre d'une communauté biotique interagissant dynamiquement. Il s'agit de penser le dehors et le dedans comme un continuum d'interaction.

Une exploration de la perception selon le principe de l'interaction entre un individu et son environnement suppose d'étudier comment l'individu reçoit les événements de son environnement mais également comment la perception est déjà une action: comment prendre en compte la dimension énactive, selon le terme de Varela, de la perception, et en conséquence comment les Somatiques sont aussi une façon d'affecter l'environnement. Autrement dit, si la séance Feldenkrais considère l'action de l'environnement comme agent de transformation du potentiel de geste du sujet, comment prend-elle en compte, en retour, ses effets sur l'environnement? La pensée de Feldenkrais nous semble, de ce point de vue, s'être arrêtée au milieu du gué: en effet - suivant en cela la psychanalyse - Feldenkrais pense bien la relation praticien/ élève (particulièrement dans la séance individuelle) comme une relation réciproque, où le praticien se laisse affecter par l'interaction autant que l'élève, se mettant à l'écoute de ses réponses, des variations de tension, de singularités de son geste afin d'alimenter le dialogue (Ginsburg, 1996). Mais cette réciprocité connaît de strictes limites: elle s'organise autour de la suprématie du praticien, et sur une idée très hiérarchisée du contrôle, toute la pratique Feldenkrais étant organisée par cet adage savoir ce que je fais pour pouvoir faire ce que je veux. Quels changements s' imposeraient aux Somatiques pour prendre en compte la question de la relation sujetenvironnement, au-delà de l'échange entre praticien et élève? Et que fait cette question à la pensée somatique? Telles sont les questions soulevées par la mise en dialogue des Somatiques et de l'écologie, et qui demandent à être entreprises si les acteurs des Somatiques veulent réellement revendiquer une éco-fonction de leurs pratiques. 


\section{Notes}

${ }^{1}$ Maurice Merleau-Ponty (1908-1961), philosophe français dont l'oeuvre est traditionnellement rattachée à la phénoménologie, a produit des travaux qui font référence dans le domaine de la perception et de la définition du monde du sentir.

${ }^{2}$ Traduit de l'original en anglais: "Plants in general are not animate; they do not move about, they do not behave, they lack nervous system, and they do not have sensations. In these respects they are like the objects of physics, chemistry, and geology" (Gibson, 1986, p. 7).

${ }^{3}$ Les trois grands courants actuels des éthiques environnementales s'éloignent tous d'un anthropocentrisme primaire. Nous ne partageons pas l'anthropocentrisme élargi, c'est pourquoi nous proposons de travailler ici indistinctement sur les éthiques environnementales individualistes (bio-centriques) ou communautaire et holiste (éco-centrisme) (Larrère, 2002).

${ }^{4}$ Pour une analyse des références scientifiques dans les écrits de Moshe Feldenkrais, voir Clavel (2015).

${ }^{5}$ Bonnie Bainbridge Cohen est la fondatrice du Body-Mind Centering ${ }^{\circledR}$, une pratique somatique qui fait une large place à la notion d'anatomie expérientielle. Elle enseigne aujourd'hui encore aux Etats-Unis et dans de nombreux autres pays.

${ }^{6}$ Pour être plus précis, il ne s'agit pas ici de penser un répertoire des variations présentes au sein d'une espèce. Certains traits, comme la couleur des yeux, sont en effet variables, mais par contre les yeux humains ne seront jamais vert fluo ou rose bonbon, le domaine des possibles n'explorant pas ces différentes expressions. À l'inverse, le plan d'organisation de tous les tétrapodes est d'avoir quatre membres, et l'environnement étant toujours moteur du changement, ce trait ne varie pas sauf conditions environnementales exceptionnelles, comme celles présentes autour des centrales de Tchernobyl ou de Fukushima. En effet, pendant la période du développement embryonnaire, l'organisme interagit avec son environnement et l'un des effets de la radioactivité est de dégrader les mécanismes de réplication et l'AND, provoquant ainsi un fort taux de mutations.

${ }^{7}$ Leigh Van Valen (1935-2010), biologiste américain (Université de Chicago) travaillant sur les théories évolutives, et auteur de la Théorie de la Reine Rouge.

${ }^{8}$ Bien que nous soyons conscientes des différences d'échelles de temps, le modèle de la Reine Rouge nous paraît cependant pertinent pour penser l'interaction qui reste mouvante et changeante au moment présent.

${ }^{9}$ Un des rares auteurs à rendre compte de cette question est $\mathrm{D}$. H. Johnson qui relate les difficultés rencontrées lors de la prise en charge de deux patients issus de cultures différentes (Johnson, 1994).

${ }^{10}$ Ce sont principalement les commentaires de L'Almanach d'un Comté des Sables d'Aldo Leopold qui sont à la base de tout le courant des éthiques environnementales. 


\section{Références}

BOTTIGLIERI, Carla. Soigner l'Imaginaire du Geste: pratiques somatiques du toucher et du mouvement. Chimères - soigne qui peut (la vie), Toulouse, Erès, n. 78, p. 113-128, fév. 2013.

BOTTIGLIERI, Carla. Médialités: quelques hypothèses sur les milieux de Feldenkrais. Dans: GINOT, Isabelle (Dir.). Penser les Somatiques avec Feldenkrais: politiques et esthétiques d'une pratique corporelle. Laveyrune: L'Entretemps, 2015. À paraître.

BOTTIGLIERI, Carla; GINOT, Isabelle; SALVATIERRA, Violeta. Du Bien-être comme Devenir Subjectif: techniques du corps et techniques de soi. Dans: FLORIN, Agnès; PREAU, Marie (Dir.). Le Bien-être. Paris: L'Harmattan, 2013. P. 243-254. (Collection Logiques Sociales.)

CARROLL, Lewis. Alice au Pays des Merveilles. Traduction: Henri Bué. Londres: Macmillan, 1869.

CLAVEL, Joanne. Les Sciences dans les Ecrits de Feldenkrais. Dans: GINOT, Isabelle (Dir.). Penser les Somatiques avec Feldenkrais: politiques et esthétiques d'une pratique corporelle. Laveyrune: L'Entretemps, 2015. À paraître.

DARWIN, Charles. L'Origine des Espèces. Traduction: Edmond Barbier. Paris: La Découverte, 1985 [1859].

FOSTER, Susan. Choreographing Empathy: kinesthesia in performance. New York: Routledge, 2011.

GIBSON, James. The Ecological Approach to Visual Perception. New York: Taylor \& Francis Psychological Press, 1986 [1979].

GINOT, Isabelle. Douceurs Somatiques. Repères, Cahiers de Danse, Val-de-Marne, La Briqueterie, n. 32, p. 21-25, fév. 2013.

GINSBURG, Carl. Is there a Science to the Feldenkrais Magic? Feldenkrais Research Journal, Paris, International Feldenkrais Federation (IFF), Rapport, s. v., p. 7-12, 1996.

HANNA, Thomas. What is Somatics? Dans: JOHNSON, Don Hanlon (Dir.). Bones, Breath and Gesture. Berkeley: North Atlantic Books, 1995. P. 139-152.

JOHNSON, Don Hanlon. Body, Spirit and Democracy. Berkeley: North Atlantic Books \& Somatic Resources, 1994.

LARRÈRE, Catherine. Avons-nous Besoin d'une Ethique Environnementale. Dans: COLLIN, Jean-François (Dir.). Cosmopolitiques 1: la nature n'est plus ce qu'elle était. Paris: L'Aube, 2002. P. 69-85.

LEOPOLD, Aldo. Sand Country Almanach. Oxford: Oxford University Press, 1949.

LEWONTIN, Richard. La Triple Hélice: les gènes, l'organisme, l'environnement. Traduction: Nicolas Witkowski. Titre original: Gene, Organismo e Ambiente. Paris: Seuil, 2003 [1998]. 


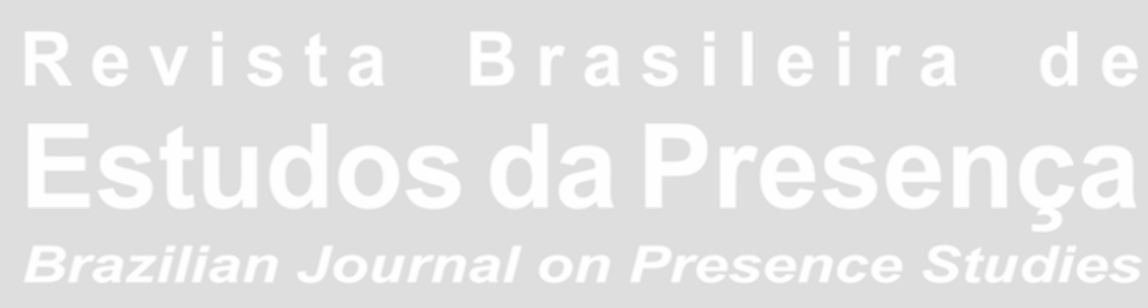

ISSN 2237-2660

MALABOU, Catherine. Les Nouveaux Blessés: de Freud à la neurologie, penser les traumatismes contemporains. Paris: Bayard, 2007.

MALLER, Cecily et al. Healthy Nature Healthy People: 'contact with nature' as an upstream health promotion intervention for populations. Health Promotion International, v. 21, n. 1, p. 45-54, 2005.

NANCY, Jean-Luc. Au Fond des Images. Paris: Galilée, 2003.

SOMATIC Approaches to Movement - interviews wih founders, teachers and choreographers. Mise en scène: Lila Greene. Entretien: Sylvie Fortin. France: La Recherche en Mouvement, s.d. 1 DVD, (127 min.). Disponible sur: <http://www.rechercheenmouvement.org>. SONIGO, Pierre; STENGERS, Isabelle. L'Évolution. Courtabœuf: EDP Sciences, 2003.

Isabelle Ginot est titulaire d'un doctorat en arts du spectacle, Professeur d'université au département Danse de l'université de Paris-VIII, et responsable du D.U. Techniques du Corps et Monde du Soin. Co-fondatrice de l'Association d'Individus en Mouvements Engagés (AIME), elle développe avec l'association un ensemble de pratiques corporelles issues de la danse et des pratiques somatiques, en direction de personnes en situation de précarité sociale ou de santé. Elle est praticienne certifiée de la méthode Feldenkrais. La plupart de ses publications sont accessibles sur le site www.danse.univ-paris8.fr.

E-mail: isabelle.ginot@univ-paris8.fr

Joanne Clavel est diplômée d'un doctorat en Écologie et Évolution de l'Université Paris 6. Chercheuse associée au département Danse de Paris 8, ses recherches sur les liens entre pratiques corporelles et Ecologie s'orientent dans des perspectives éthiques, esthétiques et politiques. Elle a fondé le collectif d'artistes Natural Movement afin de monter des projets artistiques de médiation sur la biodiversité et ainsi, développer une éco-culture pour le futur. Elle conduit ses recherches au Muséum National d'Histoire Naturelle de Paris.

E-mail: joanne.clavel@gmail.com

Ce texte inédit, révisé par Annelyse Gayraud, est également publié en portugais dans ce numéro. 